\section{Transient \\ Gratings in Semiconductors}

\section{J. Vaitkus, K. Jarašiūnas}

Department of Semiconductor Physics \& Institute of Materials Science and Applied Research, Vilnius University, Lithuania.

\section{The large variety of optical non-linearities that arises in semiconductors are leading to the development of many techniques based on transient gratings for measuring several different types of properties.}

The diffraction of light by a nonpermanent grating was first demonstrated using a grating-like structure induced by ultrasonic waves that were passed into a crystal. The invention of the laser with its extraordinary coherence then made it possible to use the interference between light waves to create transient gratings in various media such as solids, liquids with saturable absorption, and gases in which resonances were excited. These lightinduced gratings aroused considerable interest in various areas of laser-related research, and they eventually led to methods for investigating optically excited materials $[1,2]$.

\section{Light-induced Diffraction}

Much of the early work on real-time holography in silicon crystals [3] stimulated interest in light-induced diffraction and in the mechanisms responsible for modulation of the optical properties of bulk crystals. Light-induced diffraction eventually emerged as a non-destructive technique for monitoring the dynamics of nonequilibrium plasma which could be used to investigate the generation, diffusion and recombination of charge carriers in semiconductors [4]. Pioneering work on transient gratings in semiconductors stimulated the development of the degenerate four-wave mixing (DFMW) technique as a method for "active" spectroscopy, where the principles of optical holography ensured high sensitivity, visualisation of the modulation of the refractive index and real-time measurements of the dynamics.

Experimental techniques based on light-induced transient gratings are illustrated in Fig. 1 showing the optical paths for different types of measurements. Two coherent laser beams interact within the sample to excite an interference pattern. The pattern has an intensity $I$ as a function of the lateral distance $\mathrm{x}$ within the sample given by $I=I_{\mathrm{o}}(1+\cos 2 \pi x / \Lambda)$, where $I_{\mathrm{o}}$ is the incident intensity and $\Lambda$ the spacing of the interference peaks. Free carriers generated at the maxima of the interference field induce changes in the electrical properties. These changes are monitored in the DFMW method by observing the sample's optical properties using the intensities of the zero- or firstorder beams resulting from diffraction of a low-intensity probe beam. The change in beam polarization can be monitored by inserting a prism in the first-order diffracted beam.

Modulation of the index of refraction and/or absorption by the carrier plasma takes place via different mechanisms. These range from resonant effects at the band gap to refraction determined solely by modulation of the carrier concentration at energies well below the band-gap energy. Non-linear optical properties induced by the generation of a carrier plasma owing to transitions between the valence and conduction bands have been widely observed in many semiconductors (e.g., Si CdSe, GaAs and CdTe excited by a Nd:YAG $1.064 \mu \mathrm{m}$ pulsed laser ).

The Drude-Lorentz theory predicts that a concentration $N$ of light-induced free carriers leads to a periodic modulation $\Delta n$ of the refractive index $\mathrm{n}$ given by:

$$
\Delta n=-\mathrm{e}^{2} N / 2 n m^{*} \omega^{2} \varepsilon_{\mathrm{o}}
$$

for an effective mass $m^{*}$ of the electron hole pair, a modulation depth $\varepsilon_{\mathrm{o}}$, and a laser of frequency $\omega$ and wavelength $\lambda$. Spatial modulation of the electrical properties therefore generates temporary changes in the optical properties which can be observed by light diffraction from the modulated structure.

For semiconductors, light-induced changes of the refractive index usually dominate in the case of non-resonant excitation. The diffraction efficiency $\eta$ of the transient gratings which arise is related in a simple way to the incident $I_{\mathrm{do}}$ and dif- fracted $I_{\mathrm{d} 1}$ beam intensities by the lightinduced change of the refractive index. For the case of a grating in a thin sample of thickness $h$,

$$
\eta=I_{\mathrm{d}} / I_{\mathrm{do}}=4 \pi^{2}(\Delta n h)^{2} / \lambda^{2}
$$

where the non-equilibrium carrier concentration is given by Eq. (1).

The nonequilibrium carriers diffuse and recombine so the diffraction efficiency of the grating changes with time. The grating time constant $\tau_{\mathrm{g}}$ is given by

$$
1 / \tau_{\mathrm{g}}=1 / \tau+4 \pi^{2} D / \Lambda^{2}
$$

where $\tau$ is the lifetime and $D$ the diffusivity of the free carriers and $\Lambda$ is the spacing of the grating. Plotting $1 / \tau_{\mathrm{g}}$ as a function of $1 / \Lambda^{2}$ yields a straight line where the intercept gives the carrier lifetime and the gradient gives the diffusivity. Examples of the experimentally determined dependence of $\tau_{\mathrm{g}}$ on the spacing of the grating are plotted in Fig. 2 for three types of semiconductors.

Techniques based on light-induced transient gratings are attractive for semiconductor research because they offer: - High sensitivity, i.e., a large signal-tonoise ratio, since the diffracted probe beam is located in the dark field away from the interfering beams. Probe-beam intensities can be measured to one part per million corresponding to a $\Delta n$ of the order of $10^{-6}$ or less.

- Simple separation of carrier recombination and carrier diffusion by varying the grating period, i.e., by changing the angle between the interfering beams.

- Possibility of using pump-probe techniques to separate surface processes from

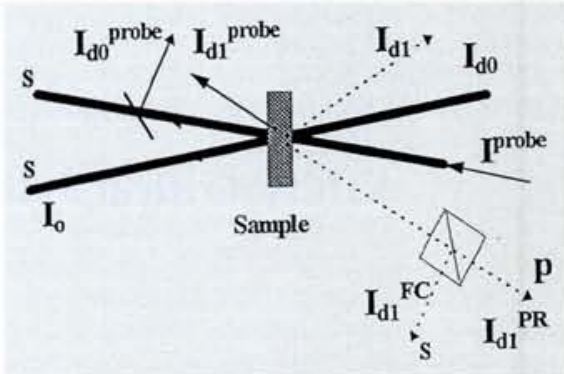

Fig. 1. A schematic illustration of the optical paths for experimental studies based on transient gratings. Two coherent laser pump beams with a given polarization interact within the sample to excite an interference pattern. The intensities, $I_{\mathrm{d} 0}$ and $I_{\mathrm{d} 1}$ of the zero- and firstorder diffracted beams are measured along with the intensities, $/ I_{\mathrm{d} 0}^{\text {poose }}$ and $I_{\mathrm{d} 1}^{\text {probe }}$, of the zero- and first-order beams produced by diffraction of a low-power probe beam. A prism mounted in the path of a first-order pump beam can be used to determine the change in polarization. 
processes occurring in the bulk. Laser beams with two different fixed wavelengths are used, where strongly absorbed beams excite a transient grating at the surface while a weakly-absorbed time-delayed probe beam monitors the decay of the grating. The diffracted signal integrates the carrier modulation depth over the sample thickness to provide information on the surface recombination velocity and bulk recombination and diffusion processes. For example, surface recombination velocities in the range $10^{4}-5 \times 10^{5}$ $\mathrm{cm} / \mathrm{s}$ have been measured in bulk GaAs and $\ln \mathrm{P}$ crystals using picosecond YAG laser pulses at $0.53 \mu \mathrm{m}$ for excitation and at $1.06 \mu \mathrm{m}$ for probing.

In general, by monitoring a laser beam diffracted by a light-induced grating one can directly obtain electronic parameters of semiconductors, such as the carrier concentration, lifetime, mobility, and rate of surface recombination using entirely optical techniques. Aside from fundamental studies, these techniques also offer a simple way to control changes in a semiconductor's technically important electronic properties resulting from doping, implantation, annealing, etc. For example, a study of the homogeneity of GaAs wafers (Fig. 3) has revealed the distribution of growth defects in wafers [6].

\section{Other Effects}

The interaction of light with matter is in fact more complicated than that described by the Drude-Lorentz theory, so mechanisms for modulating the refractive index other than that based on excess carriers have to be considered.

A high diffraction efficiency is possible in the vicinity of an absorption edge owing to resonance between laser quanta and excitons. Dynamic screening of excitons has been demonstrated as well as a temperature-dependent detuning of the exitonic resonance [7]. A coherent superposition of states can be created using tuneable dye-lasers in order to probe the evolution of the states. This has enabled the transient-grating technique to be extended into the subpicosecond time domain to study the dephasing times of free excitons, biexcitons, etc., as well as quantum beat and polarization interference phenomena in bulk crystals and quantum wells using a technique called time-resolved coherent laser spectroscopy.

The generation of free carriers also gives rise to nonlinearities related to carrier transport. Modulation of the refractive index owing to light-induced space-

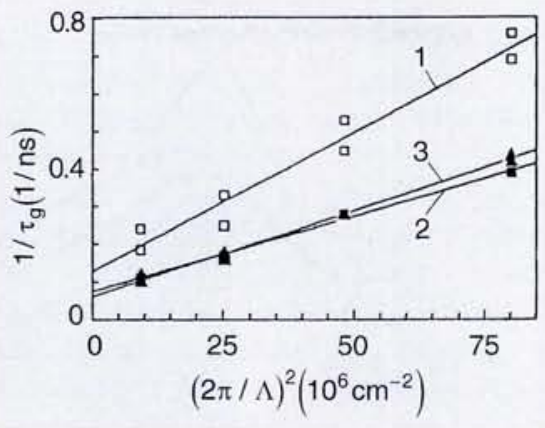

Fig. 2. The intercepts and gradients of straight lines through experimentally determined grating time constants plotted as a function of the grating spacing give estimates of the carrier lifetime and diffusivity. The experimental data are for: an InGaAs epitaxial layer on an InP substrate (1); an InGaAs epitaxial layer between two InP layers (2); an InGaAs layer on InP (3).

\section{charge electric fields - a well-known} electro-optic effect - represents an excellent example of this type of nonlinearity. These fields arise as a result of charge redistribution: nonequilibrium electrons generated at deep traps within the conduction band move to unilluminated areas by diffusion or drift to become trapped at new sites.

At short pulse times, excited free carriers and photorefractive gratings coexist, and the dynamics of the gratings on subnanosecond timescales reveal rapid charge redistribution. A photorefractive grating phase shifted by $90^{\circ}$ is formed owing to diffusion of mobile carriers with the creation of a space-charge field between the carriers and ionized deep traps. Drift of the majority carriers in the space-charge field opposes the decay of the grating by diffusion. Meanwhile, the diffusion and drift of minority carriers tends to reduce the space-charge field, and may lead to a

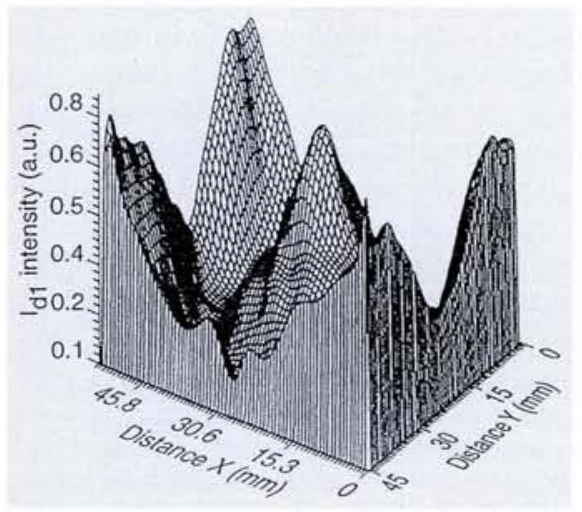

Fig. 3. A technological application of light-induced transient gratings: a map of the intensity of laser light diffracted by a $50 \times 45 \mathrm{~mm}^{2} \mathrm{GaAs}$ integrated circuit wafer reveals the presence a non-homogenous distribution of defects [6]. phase-shifted grating for the minority carriers as well as bipolar carrier diffusion at high excitations. Carrier recombination takes place at deep traps when charge transport is complete.

Although the strength of the electrooptic effect is an order smaller that the free-carrier effect, it is possible to separate the two using the anisotropy of light diffraction on photorefractive gratings (i.e., by using so-called "photorefractive cut" crystals oriented with respect to the grating vector and the polarization of the probe and diffracted beams). Studies of the coexisting gratings allow one to investigate the charge state of defects and their electrical activity (concentration), carrier lifetimes and mobilities, etc.

The availability of a tuneable infrared laser source would enable one to investigate charge redistribution between deep defects and to optimize experimental parameters for studies based on photorefractive gratings. Moreover, an enhancement of photorefractive nonlinearities may possibly arise in layered structures at the excitonic resonances, and in bulk semiconductors at excitation energies close to the energy of the band gap owing to resonant excitation.

The diffusion of hot carriers in a strong external electric field offers another example of a novel application of the transient grating technique. Hot carrier bipolar diffusion coefficients have been measured directly by combining electron heating in external microwave field with the self-diffraction of light by a freecarrier grating. The field dependence of the diffusion of the hot electrons was determined (Fig. 4), a property that is relatively difficult to measure by other methods [8].

More recent studies of carrier transport in inhomogeneous electric fields within $\mathrm{GaAs}$ and InP bulk crystals have revealed novel phenomena, notably the light-induced creation of high-field domain gratings (Gunn-type gratings) with values of the local electric field approaching $100 \mathrm{kV} / \mathrm{cm}$. The diffraction of light by this type of electro-optical nonlinearity was found to be more efficient than for free-carrier gratings [9]. This novel effect may open the way towards a purely optical technique for creating and studying the ultrafast dynamics of Gunndomain formation.

\section{Applications}

The ability to select a suitable excitation depth (by changing the wavelength of 
the pump beams), grating modulation depth (by changing the ratio of intensities of the beams) and probe beam (by operating in a suitable spectral region) mean that a wide range of parameters can be measured that are difficult to investigate using other methods.

Layered systems and superlattices are of particular interest. By generating a freecarrier grating and probing it in the spectral region lying outside the resonant interaction one can investigate free-carrier diffusion, recombination and redistribution within layers hidden beneath a transparent layer [5]. The high sensitivity is demonstrated by the fact that the influence of doped monolayers on neighbouring layers can be studied.

Moreover, the possibility to tune the excitation and probe beams over wide wavelength ranges allows one to determine the spectral properties of a bulk material or a structure. Defects in superlattices and their distribution have been analyzed by measuring recombination parameters [10], and details about defects, such as changes of the local field symmetry due to lattice deformation, can be probed with high sensitivity [11].

The analysis of effects related to free and localised carriers shows that diverse applications of the transient-grating technique are possible, in both fundamental research and applied fields. The most immediate application perhaps involves non-destructive techniques to investigate transport processes in semiconducting crystals and two-dimensional layered structures - a technique that may be suitable for the in situ control of production processes used in the electronics industry. Transient gratings excited by picosecond and nanosecond laser pulses have also been used to monitor the quality of crystals and layers as well as the annealing of defects introduced by thermal effects and by irradiation.

For instance, Vilnius University's Laboratory for Optical Diagnostics of Semiconductors has developed the DSCAN instrument to monitor the homogeneity of GaAs wafers by mapping the distribution of dislocations and trapping centres. The laboratory has also developed the IMPLANT instrument to control very low doses of implanted ions (down to concentrations of $10^{12}$ atoms $/ \mathrm{cm}^{3}$ of $\mathrm{B}^{+}, \mathrm{P}^{+}$ or $\mathrm{Ar}^{+}$) in Si wafers.

Finally, photorefractive gratings have potential applications in optoelectronics, high-speed information processing, phase-conjugated devices, real-time optical sensors, etc.

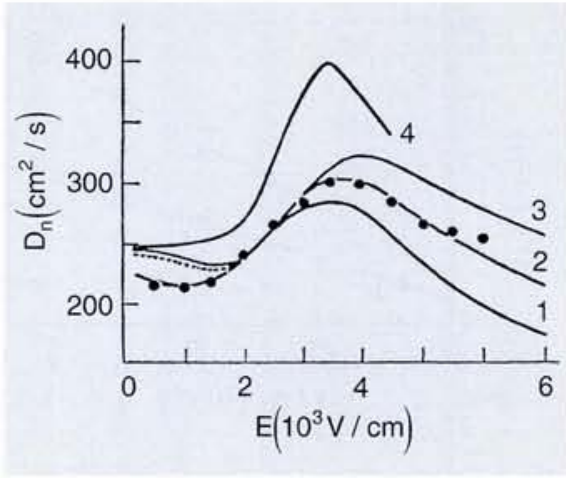

Fig. 4. The electric-field dependence of the diffusion coefficient of electrons in GaAs [8]. The solid curves correspond to Monte Carlo simulations for increasingly larger values of the coupling constant $(1: 0.18 \mathrm{GeV} / \mathrm{cm}$; 2: $0.3 \mathrm{GeV} / \mathrm{cm} ; 3: 0.5 \mathrm{GeV} / \mathrm{cm} ; 4: 1.0 \mathrm{GeV} / \mathrm{cm})$. The points correspond to optical measurements made using transient gratings. They indicate that the diffusion coefficient is smaller than the predicted value at fields below about $2 \times 10^{3} \mathrm{~V} / \mathrm{cm}$ owing to scattering on ionized impurities.

\section{Future Developments}

Non-linear optical polarizability becomes important at high laser intensities. Since this effect changes the refractive index of a crystal it may be possible to create a transient grating using a purely optical effect. Gratings of this type could be used to investigate the non-linear optical properties of semiconductors $[12,13]$.

Secondary effects related to energy transfer from the excited state to a lattice can arise in addition to free-carrier and non-linear optical effects. For instance, local heating induced in a crystal by light absorption and free-carrier recombination may lead to changes of the refractive index and the formation of a thermal grating, thus allowing the thermal conductivity to be measured using the decay of the grating [14]. Moreover, thermal expansion of the lattice can lead modulation of surface relief, with diffraction of the light reflected by the surface structure [15]. By examining time constants as well as the change in the sign of the refractive index it should be possible to separate out the various phe-

\section{Linac-Driven FEL Approved}

Linac-driven free-electron lasers (FEL) providing short-wavelength (1-10 $\AA$ ) coherent radiation with eight orders of magnitude larger peak brilliance compared to state-of-the-art synchrotron radiation sources seem to be feasible. They use self-amplified spontaneous emission whereby an electron beam of sufficient quality passing a long undulator magnet exponentially amplifies an initially existing radiation field.

DESY Hamburg's HASYLAB has been given the go-ahead to integrate a FEL in the TESLA test bed facility that is under construction [see EN 25 nomena that lead to transient gratings.

Improved experimental techniques offer many opportunities. For instance, an increased sensitivity to free and bound carriers and to excitons would permit electronic properties to be probed deep within crystals and layered structures. The development of waveguide geometries other than thin-layer structures would allow excitation laser beams of reduced intensity to be used for samples that are sensitive to high-power laser light. Other geometries may not only decrease the laser intensity threshold at which the very interesting non-linear optical effect is observed but also open up other wavelength ranges. So techniques based on transient gratings clearly have a great potential for further development and application.

\section{References}

[1] H.J. Eichler, P. Gunter \& D.W. Pohl, "LaserInduced Dynamical Gratings”, Springer Series Opt. Sci. 50 (Springer, Heidelberg; 1987).

[2] R.K. Jain, M.B. Klein in Optical Phase Conjugation (Academic Press, New York; 1983) 307. [3] J.P. Woerdman, Philips Res. Reports Suppl., (1971) No. 7.

[4] K. Jarašiūnas \& J.Vaitkus, phys.stat.sol. A 23 (1974) KL9; ibid 44 (1977) 793.

[5] J.Vaitkuset al.,. IoP Conf Ser. No. 141, Chapter 3 (IOPP, Bristol; 1995) 369.

[6] J. Vaitkus et al., Semiconductor. Sci. ¿ Techn. 7 (1992) 131.

[7] R. Baltramiejunas, J. Vaitkus \& K. Jarašiūnas, Sov. Phys.-Semicond. 10 (1976) 969.

[8] J. Vaitkus et al., Proc.19th Int. Conf. Physics of Semicond., Vol. 2 (Inst. of Physics, Polish Academy of Sci.; 1988) 1447; J. Vaitkus et al., Sov. Phys. Collect. 30 (Alerton Press; 1990) 336. [9] L. Subacius et al., Proc. SPIE 2648 (1995) 207.

[10] J. Vaitkus et al., Lithuanian J. Phys. 5 (1995) 492.

[11] K. Jarašiūnas et al., Optics Lett. 19 (1994) 1946.

[12] A. Smirl et al., J. IEEE Quant. Electr. 24 (1988) 289.

[13] E.J. Canto-Said et al., IEEE J. Quant. Electr. QE-27 (1991) 2274.

[14] H. Eichler, G. Salje \& H. Stahl, J. Appl. Phys. 44 (1973) 5383.

[15] J. Vaitkus, E. Gaubas \& K. Jarašiūnas, Soviet Phys. - Solid State 20 (1978) 3160.

(1996) 104]. A international collaboration plans is to demonstrate proof-of-principle of a $200 \mathrm{eV} / 60$ $\AA ̊$ VUV FEL by the year 2000 . Several beams could be then extracted from the linac and piped to a synchrotron users laboratory where they would be switched between several undulators.

Linac-driven $x$-ray FELs are being considered in the context of a future $500 \mathrm{GeV}, 30 \mathrm{~km}$ long high-energy linac for particle physics (the linac's first section would provide $1 \AA$ radiation). A final draft of a US design is expected shortly and a Japanese design report is due next year. Meanwhile physicists are studying opportunities offered by linac-driven FELs' exceptionally bunch brilliance. 